\title{
The origin of the chemical elements in cluster cores
}

\author{
J. de Plaa ${ }^{1, \star}$ \\ SRON Netherlands Institute for Space Research, Sorbonnelaan 2, 3584 CA Utrecht
}

Received 2 July 2012, accepted XXXX

Published online XXXX

Key words X-rays: galaxies: clusters, galaxies: clusters: general, galaxies: abundances, supernovae: general

\begin{abstract}
Metals play a fundamental role in ICM cooling processes in cluster cores through the emission of spectral lines. But when and how were these metals formed and distributed through the ICM? The X-ray band has the unique property of containing emission lines from all elements from carbon to zinc within the $0.1-10 \mathrm{keV}$ band. Using XMM-Newton, the abundances of about 11 elements are studied, which contain valuable information about their origin. Most elements were formed in type Ia and core-collapse supernovae, which have very different chemical yields. Massive stars and AGB stars also contribute by providing most of the carbon and nitrogen in the ICM. Because feedback processes suppress star formation in the cluster centre, the element abundances allow us to directly probe the star formation history of the majority of stars that are thought to have formed between $z=2-3$. The spatial distribution in the core and the evolution with redshift also provide information about how these elements are transported from the member galaxies to the ICM. I review the current progress in chemical enrichment studies of the ICM and give an outlook to the future opportunities provided by XMM-Newton's successors, like Astro-H.
\end{abstract}

Copyright line will be provided by the publisher

\section{Introduction}

After the 'Big Bang', the baryonic component of the Universe mainly consisted of hydrogen and helium with traces of lithium and beryllium. The first metals with a higher atomic weight were produced in the first generation of stars, also referred to as Population III stars, which started the epoch of re-ionization. The nature of this stellar population is very uncertain, but it likely consisted of intermediatemass and high-mass stars (Vangioni et al. 2011). Based on WMAP measurements, these stars formed around $z \sim 10$, when the age of the Universe was about 500 Myr. Although these stars were the first to enrich the surrounding gas, the total contribution to the current day chemical composition is thought to be small, only $10^{-4} Z_{\text {solar }}$ (Matteucci \& Calura 2005).

The bulk of the enrichment probably occurred around $z \sim 2-3$ through the supernova explosions following major star bursts. A compilation of the universal star formation rate measurements as a function of redshift (Hopkins \& Beacom 2006) shows that the star formation rate peaked around $z \sim 2-3$ and declined slowly to low redshift. However, these are averaged rates over both cluster and field galaxies. At a similar redshift, the Intra-Cluster Medium (ICM) in clusters of galaxies starts to form, which, together with feedback from Active Galactic Nuclei (AGN), quenched the star formation in these objects. Therefore, in the cluster of galaxies case, the star formation rate drops much faster than average (Gabor et al. 2010). In that respect, clusters of galaxies are a special environment, because their

^ Corresponding author: e-mail: j.de.plaa@sron.nl enrichment is dominated by the products from the main star bursts at $z \sim 2-3$.

Ferrara et al. (2005) have estimated that a major fraction ( $>90 \%$ ) of the produced elements around $z=3$ contributes to the enrichment of gas in a hot phase, instead of ending in newly formed stars. The ICM in clusters therefore contains the bulk of the metals produced in the cluster member galaxies. In the period of the major star bursts, the main enrichment mechanisms that were transporting the metals from the galaxies to the surrounding medium were galactic winds driven by the supernova explosions and AGN uplifting of galactic gas. At a lower level, also other enrichment mechanisms play a role. Metals can be ejected from the galaxies by galaxy-galaxy interactions, by sloshing motions of the hot ICM, and ram-pressure stripping of in-falling galaxies (see Schindler \& Diaferio 2008 for a review).

\section{Origins of elements}

The bulk of the elements heavier than beryllium are produced in the end phases of stellar evolution. The elements in the mass range between oxygen and silicon are mainly produced by core-collapse supernovae, because the elements with masses higher than silicon that are produced in the core region of the massive star are compressed into the neutron star or black hole that is formed during the supernova event. Only the outer layers containing the lighter elements are ejected into the surrounding medium. The elements from silicon to nickel, however, are the main products of type Ia supernovae, which are exploding white dwarfs in binary systems. During such a supernova event, elements up to 


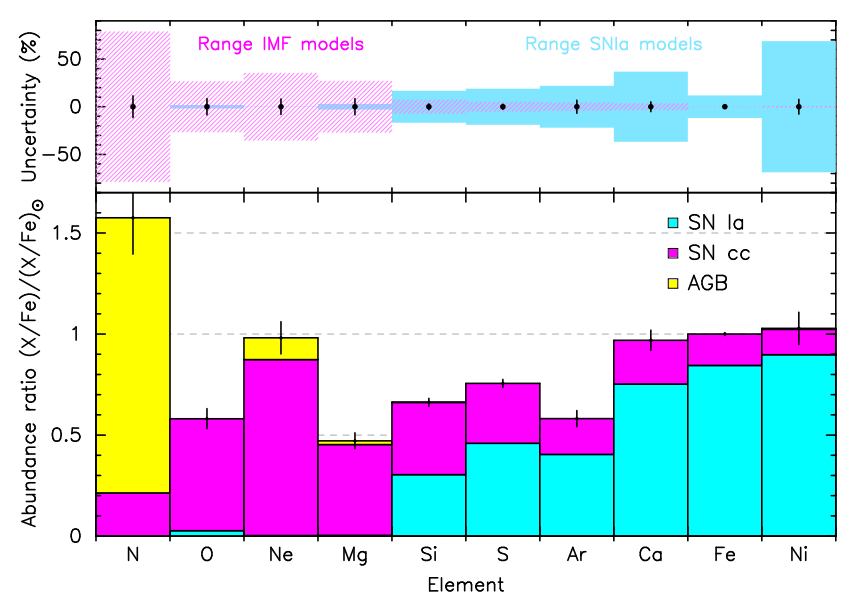

Fig. 1 Expected abundances measured in a $120 \mathrm{ks}$ XMMNewton observation of Sérsic 159-03 (bottom panel), which is a typical bright local cluster. The statistical error bars were obtained from de Plaa et al. (2006). The estimates for the SNIa, SNcc, and AGB contributions are based on a sample of 22 clusters (de Plaa et al. 2007) and two elliptical galaxies (Grange et al. 2011). The top panels show the typical range in SNIa and IMF models with respect to the statistical error bars in the observation.

nickel are produced by explosive fusion and the white dwarf is disintegrated, which releases all the products in the surrounding medium.

A few light elements, however, have a different origin. Carbon is thought to be mainly produced by massive stars during their lifetime and ejected through the stellar winds. Nitrogen is ejected by intermediate-mass stars in the Asymptotic Giant Branch (AGB) phase. Since elements heavier than nickel are not produced by fusion, and are therefore less abundant, we do not discuss the origin of these metals in this paper.

Figure 1 shows the expected contributions of the most abundant metals that can be detected using the XMM-Newton observatory. The bars indicate the relative contribution of each supernova type to the abundance of the elements. The fractions depend on the supernova models used. The effect of uncertainties in the supernova models and in the Initial-Mass Function (IMF) are indicated in the top panel.

\subsection{Type Ia supernovae}

Although type Ia supernovae are used as standard candles for cosmology, their progenitor and explosion mechanism are still poorly known. The common misconception is that a type Ia supernova occurs when a white dwarf goes over the Chandrasekhar mass. Instead, the explosive carbon fusion in the star is ignited just before it reaches this limit. In recent years, optical searches for supernovae have yielded hundreds of observations of type Ia's which revealed a surprising variety in their properties. It has proved to be very difficult to link the observed supernovae to possible progenitors (see e.g. Howell 2011 for a recent review).
Currently, roughly three main progenitors are being considered. The first is a 'classical' type Ia or also called 'Single Degenerate' (SD), where the companion of the white dwarf is a main sequence or red-giant star that accretes material on the white dwarf. The second is a scenario where two white dwarfs merge and the less massive white dwarf is accreted onto the more massive one, which is known as the 'Double Degenerate' (DD) scenario. And the last is a sub-Chandrasekhar channel, where a thick layer of helium builds up on the white dwarf's surface either by hydrogen burning or by a helium-rich donor. Because of the lower density of the last systems, they are thought to produce more intermediate-mass elements like silicon, sulfur, and calcium than during the deflagration in the typical SD and DD cases.

Observations have shown that relatively luminous type Ia supernovae tend to occur in spiral galaxies, while the sub luminous are mainly found in elliptical galaxies with old stellar populations (e.g. Howell 2001; Sullivan et al. 2006). This appears to indicate that the brighter 'prompt' supernovae explode early (<400 Myr) after the star burst, while the sub-luminous occur with a delay of a few Gyr. This can be explained in the DD scenario by the fact that it takes a longer time to evolve a star into a low-mass white dwarf compared to a high-mass white dwarf. The delay time explanation is also supported by high-redshift studies that show that at $z=1$ type Ia supernovae are $12 \%$ more luminous and have less intermediate mass elements in their spectra than local SNIa's (e.g. Howell et al. 2007; Sullivan et al. 2009). However, also differences in initial metallicities or other unknown parameters could play a role.

One of the few successes to combine theory and observations in this field is the explanation of the observed DelayTime Distribution (DTD) using stellar population synthesis studies. Supernova rate measurements in elliptical galaxies (and clusters of galaxies) show that the rate declines with $\sim t^{-1}$ after the initial star burst. Model calculations show the same level of decline for DD mergers, which is an indication that this channel is dominating the SNIa rate at larger delay times (see Fig 2] Ruiter et al. 2009; Mennekens et al. 2010).

Due to the uncertainty in the nature of the SNIa progenitor and the poorly understood physics of the explosion (during the explosion, the burning front probably switches from being subsonic to supersonic), there is a large range of supernova Ia models predicting the metal yields. Well known examples are Iwamoto et al. (1999) and models by Bravo et al. (2012). The predicted yields for various elements can vary substantially for every model, which means that accurate abundance measurements can help to constrain the SNIa models.

\subsection{Core-collapse supernovae}

Although there is also a range of different models for corecollapse supernova yields in the literature (e.g. Woosley \& Weaver, 1995; Tsujimoto et al., 1995; Chieffi \& Limongi, 


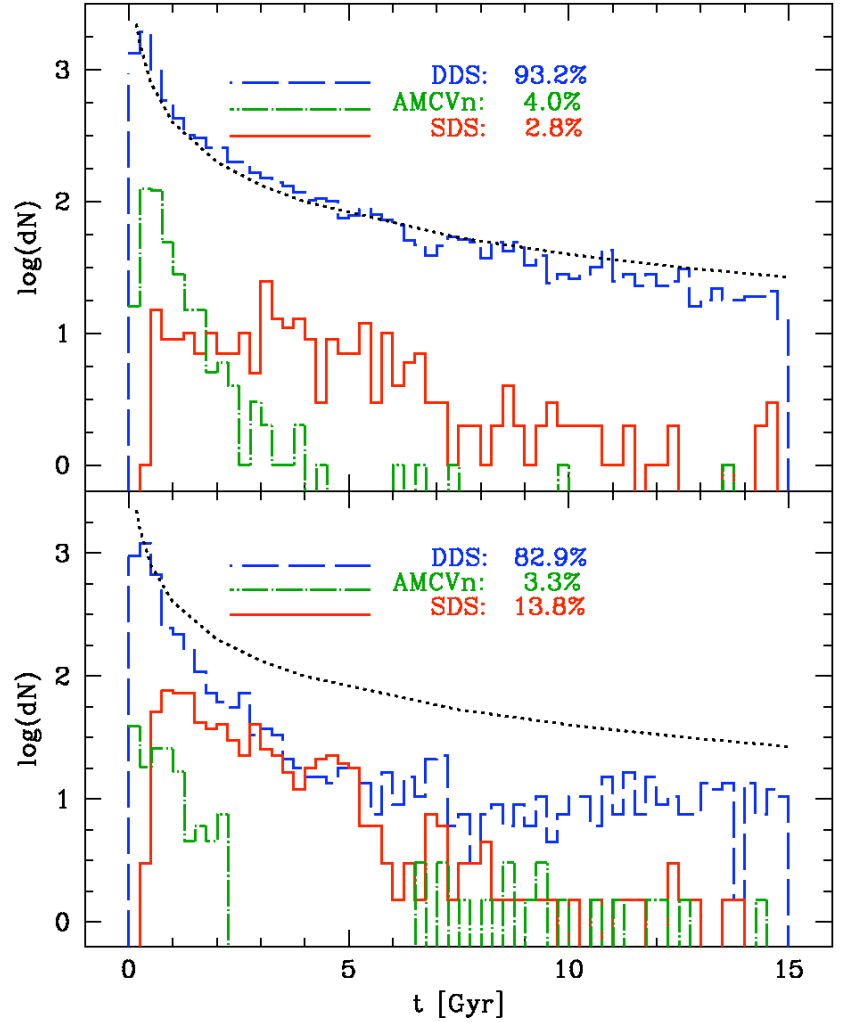

Fig. 2 Supernova type Ia delay time distributions for three different progenitor channels: white-dwarf mergers (DDS), Single-Degenerate Systems (SDS), and AM CVn systems as described in Ruiter et al. (2009). AM CVn systems are ultra-compact systems with a WD accretor and a He-rich donor. The top and bottom panel represent the result from two choices of initial parameters, but both are calculated for stellar populations in elliptical galaxies. The dotted line shows the observed decline of the type Ia supernova rate of $\sim t^{-1}$, where $t$ is the time passed since the initial star burst. The decline is consistent with the DDS progenitor model.

2004), the main parameters that determine the abundances measured are initial metallicity and the choice of the IMF. In order to determine the total contribution of core-collapse supernovae to the cluster enrichment, the model yields for each mass bin need to be weighted with the IMF in the following way (Tsujimoto et al, 1995):

$$
M_{i}=\frac{\int_{10 \mathrm{M}_{\odot} \odot}^{50 \mathrm{M}_{\odot}} M_{i}(m) m^{-(1+x)} \mathrm{d} m}{\int_{10 \mathrm{M} \odot}^{50} \mathrm{M}^{-(1+x)} \mathrm{d} m},
$$

where $M_{i}(m)$ is the $i$ th element mass produced in a mainsequence star of mass $m$. A standard Salpeter IMF corresponds to $x=1.35$, but this equation can, of course, be altered to represent other IMFs.

\subsection{Intermediate-mass AGB stars}

Since intermediate-mass stars in their AGB phase are a major source of nitrogen, it is important to include them in the

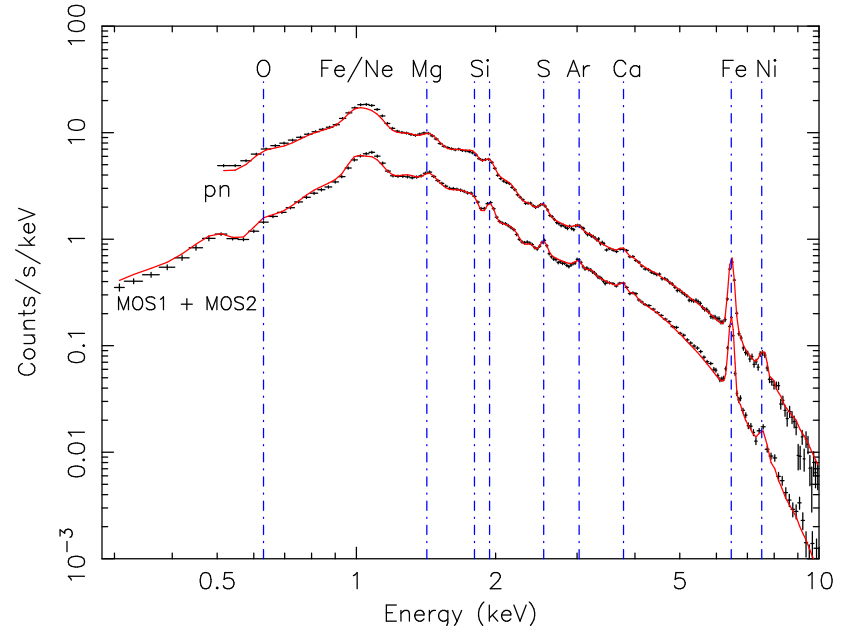

Fig. 3 EPIC spectrum of the cluster 2A 0335+096 with an exposure time of $130 \mathrm{ks}$. Adapted from Werner et al. (2006b).

model as well. Since the yields depend on the initial mass of the main sequence star, we need to weigh the yields also with the IMF like in Equation 1 AGB star yields for different masses and initial metallicities are calculated by, for example, Karakas (2010). Together with the information from the core-collapse models, the AGB stars provide an additional constraint to the initial metallicity and IMF of the stellar population.

\section{Abundance measurements in X-rays}

The soft X-ray band between 0.1 and $10 \mathrm{keV}$ is very suitable to measure abundances in hot plasmas, because it contains spectral lines of all elements from carbon to nickel. The hot ICM in clusters is particularly interesting, because the plasma is in collisional-ionization equilibrium, which simplifies the determination of the abundances. Moreover, the cluster ICM contains the integrated yield of billions of supernovae, providing a general picture of supernova yields, contrary to studies of a few individual supernovae through their remnants in our galaxy. The sensitivity of XMM-Newton, and also Suzaku, allows us to measure abundances with enough accuracy to constrain supernova models. An example of a deep EPIC observation of the cluster 2A 0335+096 in Figure 3 shows the position and strength of the lines of the most abundant elements. The availability of these excellent data sets has triggered a number of successful abundance studies (see also the review by Werner et al. 2008).

\subsection{Enrichment in local clusters}

The first attempt to link measured abundances in the hot ICM in local clusters $(z<0.2)$ was performed using the ASCA satellite (Mushotzky et al., 1996). Its instruments allowed the accurate detection of $\mathrm{O}, \mathrm{Ne}, \mathrm{Mg}, \mathrm{Si}, \mathrm{S}, \mathrm{Ar}, \mathrm{Ca}, \mathrm{Fe}$, and Ni for the first time. From this and later ASCA studies 


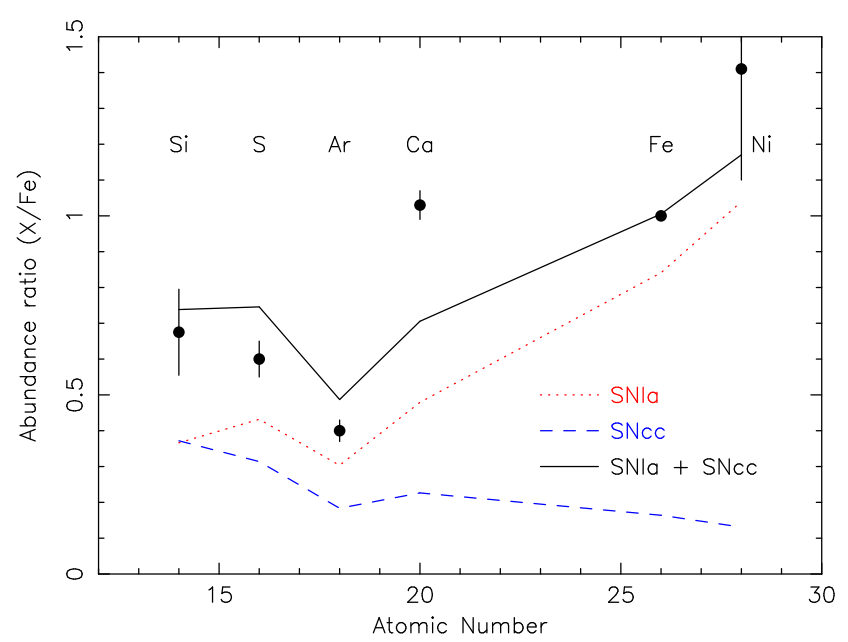

Fig. 4 Abundance ratios fitted with supernova yields from the WDD2 SNIa model (Iwamoto et al. 1999) and a SNcc model with an initial metallicity $Z=0.02$ and a Salpeter IMF. The calcium abundance appears to be underestimated. It can not explain the $\mathrm{Ar} / \mathrm{Ca}$ ratio measured in this XMMNewton sample of 22 clusters. (Adapted from de Plaa et al. 2007).

(e.g. Fukazawa et al. 1998; Finoguenov et al. 2000) a general picture emerged of an ICM which was enriched early in the cluster evolution by core-collapse supernova and later by delayed type Ia supernovae.

When more deep observations of clusters with XMMNewton became available, de Plaa et al. (2007) performed an abundance study in a sample of 22 clusters with EPIC. With the obtained accuracy, it was possible to estimate the relative contributions of type Ia and core-collapse supernova to the enrichment of the cluster by linearly combining the supernova yields for each type. For the most commonly used type Ia models (Iwamoto et al. 1999), this results in a SNIa contribution of about $30 \%$, which is remarkably similar to the $\mathrm{SNIa} / \mathrm{SNcc}$ ratio of $\sim 0.2-0.4$ as observed in the optical band (e.g. Horiuchi et al. 2011) given the fact that de Plaa et al. (2007) ignored galactic evolution effects. Surprisingly, the calcium abundance did not fit the Iwamoto et al. (1999) models well (see Fig 4), while a model by Bravo et al. (1996), which also fitted to the Tycho supernova remnant (Badenes et al. 2006), resulted in a much better fit of the Ar/Ca ratio (de Plaa et al. 2007). This showed that X-ray spectra enable us to constrain type Ia supernova models.

Several groups have performed similar studies with XMMNewton and Suzaku data since then, but usually on fewer clusters and with varying numbers of elements. In a small sample, Sato et al. (2007) performed the same fit as de Plaa et al. (2007) using abundances measured with Suzaku XIS. Unfortunately, argon and calcium were not included in the fit, but their main conclusion was consistent with de Plaa et al. (2007) considering the uncertainty introduced by the limited number of measured elements. Recently, Bulbul et al. (2012) used a modified APEC model that is able to fit the supernova type Ia to core-collapse ratio directly to the spectra. They also report a type Ia contribution of 30-40\%, which is consistent with previous work and optical data. It should be noted that this ratio depends highly on the assumed supernova models (De Grandi \& Molendi, 2009), but it is reassuring that different groups find similar numbers independently.

\subsection{The abundance of nitrogen and carbon}

In recent years, deep XMM-Newton observations of giant elliptical galaxies also allowed the detection of carbon and nitrogen. These two elements are not produced in large quantities in core-collapse supernovae, but in a variety of sources. The main origins of these elements are still subject of debate (see e.g. Romano et al. 2010), but likely candidates are metal-poor massive stars and intermediate-mass stars in their AGB phase.

Since carbon and nitrogen have a relatively low atomic mass, their K-shell transitions are located in the soft X-ray band below $0.5 \mathrm{keV}$. It also causes the lines to be stronger at lower temperatures $<1 \mathrm{keV}$ (at temperatures of a few $\mathrm{keV}$, a larger fraction of the carbon and nitrogen is fully ionised and not producing line emission). To detect the lines, it is best to observe cool elliptical galaxies with the Reflection Grating Spectrometer (RGS) aboard XMM-Newton. This instrument has sufficient effective area at low energies and a high spectral resolution to resolve the lines of these elements. Unfortunately, noise in the RGS CCD in the band where the carbon line is located prevents an accurate carbon measurement for weak sources.

Werner et al. (2006a) and Grange et al. (2011) analysed RGS data of the giant elliptical galaxies M87 (Werner), NGC 5044, and NGC 5813 (Grange) and detected nitrogen. They found that the nitrogen abundances in these objects are indeed much higher than expected based on supernova models. From the high N/O ratio, it can be deduced that nitrogen should still be produced in low- and intermediate-mass stars contrary to supernovae, because the star-formation rate in elliptical galaxies has declined very rapidly after the star bursts around $z \sim 2-3$.

\subsection{Intermediate redshift clusters}

Studying the chemical enrichment of clusters above $z=0.2$ becomes increasingly difficult, because of the lower flux of the sources. The Fe-K feature is usually the only feature strong enough to resolve at higher redshifts. Therefore, studies at these large distances focus on the iron abundance evolution as a function of redshift. In a sample of 56 clusters with redshifts in the $0.3<z<1.3$ range observed with XMM-Newton and Chandra, Balestra et al. (2007) found a significant decreasing trend of the iron abundance with redshift from roughly 0.4 solar at $z=0.3$ to 0.2 solar at $z=1.3$. It was, however, also clear that the scatter on the measured data points was relatively large. In a different sample of 39 clusters observed with XMM-Newton, Baldi 


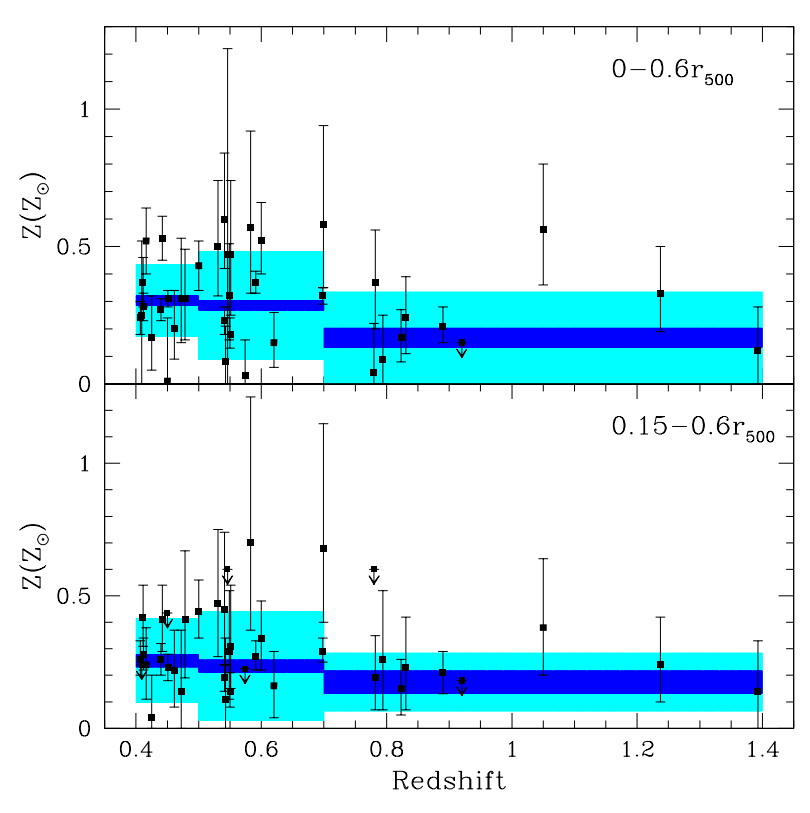

Fig. 5 Measured abundances versus redshift for a sample of intermediate redshift clusters. The top panel shows the abundances measured up to $0.6 \mathrm{R}_{500}$ and the bottom panel the results when the core of the cluster is ignored. Adapted from Baldi et al. (2012).

et al. (2012) recently reported that they could not confirm the trend found by Balestra et al. (2007), because of the high level of scatter in the data points (see Figure 5). Removing the cool cores in the spectral analysis just had a limited effect on the amount of scatter and did not allow them to draw firm conclusions. Since these measurements still only sample the tail of the SNIa delay-time distribution that originates at $z=2-3$, a strong trend may not be expected. Clearly, instruments with a much higher effective area are needed to reduce the statistical errors on these measurements.

\section{Discussion and future prospects}

X-ray spectroscopy of clusters of galaxies has proved to be a useful tool to study the enrichment history of the hot ICM, but the field is still in its early stages of development. Using current instruments, it is possible to measure the abundance of about 10 elements with an accuracy of 20-30\% if one includes systematic errors (De Grandi \& Molendi, 2009). This appears to be a substantial systematic error, however, the uncertainty in the supernova yields, given by the spread in supernova models, is sometimes more than a factor of two for certain elements. This means that even with these systematic uncertainties, the measurements can put constraints on supernova models and the IMF.

There are two main sources of systematic errors in abundance measurements: the atomic data in spectroscopic codes and instrument calibration issues. A third one can be intro-

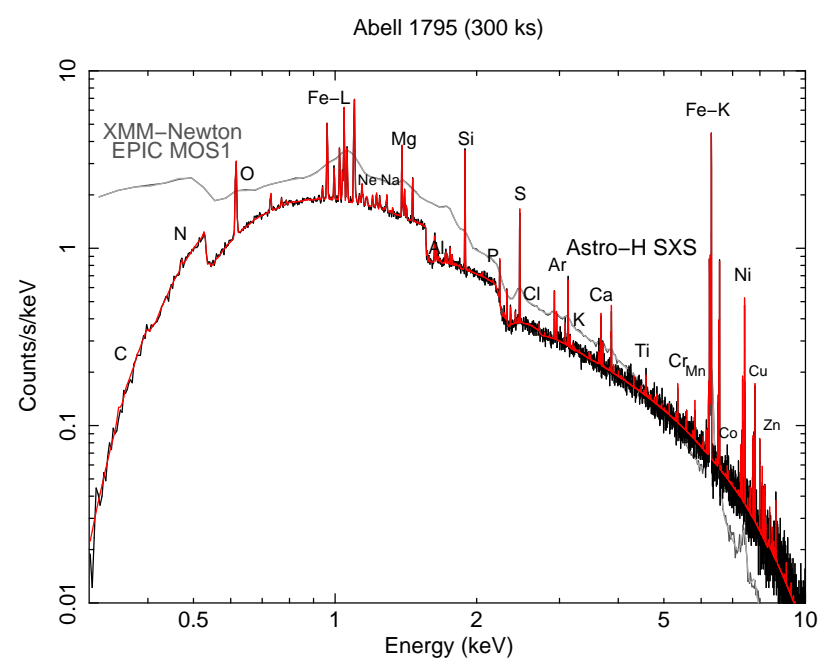

Fig. 6 Simulated Astro-H micro-calorimeter spectrum of Abell 1795 compared to an XMM-Newton EPIC MOS spectrum. The spectra were calculated using an updated version of the atomic database in SPEX including trace elements, like $\mathrm{Cl}, \mathrm{K}, \mathrm{Ti}, \mathrm{Cr}$, and $\mathrm{Mn}$.

duced when the multi-temperature structure in the gas is not taken properly into account in the spectral fit (Buote $\&$ Fabian 1998). Both the systematics in the atomic data and the calibration depend very much on the specific transition and wavelength of the line. The silicon abundance, for example, is mainly determined by measuring the Si XIV lines at about $2.0 \mathrm{keV}$, which is very close to the Au-edge of the mirror and Si-edge of the detector. This is a very challenging wavelength band to calibrate accurately. In this case, the Si XIV lines are well characterized in the atomic database, while there are also other lines from, for example, Fe XVII that have a much larger uncertainty in their oscillator strengths (de Plaa et al., 2012). It is very important to understand the systematics for each measured element individually to assess the quality of the supernova fits.

In general, most of the measured abundances have a systematic uncertainty that is lower than or not much higher than $20 \%$, which is still much better than the scatter in the models. To beat down these systematic errors, investments have to be made in the development of spectral codes and their underlying atomic data. In addition, a high level of calibration accuracy is to be pursued to improve the abundance accuracy both for current and future missions.

\subsection{Future prospects}

The next leap forward will be made using micro-calorimeter technology, which will allow spatially resolved high-resolution X-ray spectroscopy of extended sources. This type of sensor, which will be flown on Astro-H (Takahashi et al. 2010), has a typical resolution of a few eV across the soft X-ray band. With this technology, it will be possible to resolve lines from less abundant elements, like sodium, chromium, and manganese (see Fig 6). Knowledge about 
the abundances of these trace elements puts additional constraints on supernova models. The Mn/Cr ratio, for example, appears to be very sensitive to the metallicity of the type Ia progenitor (Badenes et al. 2008) and the $\mathrm{Na}$ abundance is, like nitrogen, also correlated with the contribution of intermediate mass AGB stars and sensitive to the IMF.

To measure trace elements like chromium and manganese in high resolution spectra, the spectral fitting codes that are used to fit the spectra need updates to their atomic data. Until recently, only the lines from elements with a high abundance (typically with even atomic numbers) where taken into account in the codes. The commonly used databases like ATOMDB1 (Smith et al. 2001) and the SPEX line database (Kaastra et al. 1996) are being updated and are (partly) available in the spectral fitting codes. The systematic errors in the atomic data, which can reach the $20 \%$ level in some important lines, remain an issue. Further investments in laboratory measurements of lines and theoretical line calculations are needed to improve the accuracy.

Until now, the measured abundances have been compared to individual supernova models. It would, however, be much more realistic to estimate the expected abundances from advanced binary population synthesis codes. Using these codes, it is possible to give the proper weight to the different type Ia progenitor scenarios and their respective yields. The high calcium abundance measured by de Plaa et al. (2007), for example, might be explained by sub-Chandrasekhar type Ia's, because explosive helium fusion produces more intermediate mass elements, like calcium. Predicted abundances using these detailed binary population synthesis can be directly compared to the measured abundances in clusters. The high amount of measurable elements will substantially improve the quality of the test and therefore also our knowledge of chemical enrichment in clusters.

Acknowledgements. The author likes to thank Jelle Kaastra for carefully reading the manuscript and useful discussions. SRON Netherlands Institute for Space Research is supported financially by NWO, the Netherlands Organisation for Scientific Research.

\section{References}

Badenes, C., Borkowski, K. J., Hughes, J.P., Hwang, U., Bravo, E.: 2006, ApJ 645, 1373

Badenes, C., Bravo, E., Hughes, J. P.: 2008, ApJ 680, L33

Baldi, A., Ettori, S., Molendi, S., Balestra, I., Gastaldello, F., Tozzi, P.: 2012, A\&A 537, A142

Balestra, I., Tozzi, P., Ettori, S., Rosati, P., Borgani, S., Mainieri, V., Norman, C., Viola, M.: 2007, A\&A 462, 429

Bravo, E., Martínez-Pinedo, G.: 2012, PhRvC 85(5), 055805

Bravo, E., Tornambe, A., Dominguez, I., Isern, J.: 1996, A\&A 306, 811

Bulbul, E., Smith, R. K., Loewenstein, M.: 2012, ApJ 753, 54

Buote, D. A., Fabian, A. C.: 1998, MNRAS 296, 977

Chieffi, A., Limongi, M.: 2004, ApJ 608, 405

de Grandi, S., Molendi, S.: 2009, A\&A 508, 565

\footnotetext{
1 http://www.atomdb.org

2 http://www.sron.nl/spex
}

de Plaa, J., Werner, N., Bleeker, J. A. M., Vink, J., Kaastra, J. S., Méndez, M.: 2007, A\&A 465, 345

de Plaa, J., Werner, N., Bykov, A. M., Kaastra, J.S., Méndez, M., Vink, J., Bleeker, J.A. M., Bonamente, M., Peterson, J. R.: 2006, A\&A 452, 397

de Plaa, J., Zhuravleva, I., Werner, N., Kaastra, J. S., Churazov, E., Smith, R. K., Raassen, A. J. J., Grange, Y. G.: 2012, A\&A 539, A34

Ferrara, A., Scannapieco, E., Bergeron, J.: 2005, ApJ 634, L37

Finoguenov, A., David, L. P., Ponman, T. J.: 2000, ApJ 544, 188

Fukazawa, Y., Makishima, K., Tamura, T., Ezawa, H., Xu, H., Ikebe, Y., Kikuchi, K., Ohashi, T.: 1998, PASJ 50, 187

Gabor, J. M., Davé, R., Finlator, K., Oppenheimer, B. D.: 2010, MNRAS 407, 749

Grange, Y. G., de Plaa, J., Kaastra, J.S., Werner, N., Verbunt, F., Paerels, F., de Vries, C. P.: 2011, A\&A 531, A15

Hopkins, A. M., Beacom, J. F.: 2006, ApJ 651, 142

Horiuchi, S., Beacom, J. F., Kochanek, C. S., Prieto, J. L., Stanek, K.Z., Thompson, T. A.: 2011, ApJ 738, 154

Howell, D. A.: 2001, ApJ 554, L193

Howell, D. A.: 2011, Nature Communications 2, 350

Howell, D.A., Sullivan, M., Conley, A., Carlberg, R.: 2007, ApJ 667, L37

Iwamoto, K., Brachwitz, F., Nomoto, K., Kishimoto, N., Umeda, H., Hix, W. R., Thielemann, F. K.: 1999, ApJS 125, 439

Kaastra, J. S., Mewe, R., Nieuwenhuijzen, H.: 1996, SPEX: a new code for spectral analysis of X \& UV spectra. In Yamashita, K., Watanabe, T. (Eds.), UV and X-ray Spectroscopy of Astrophysical and Laboratory Plasmas (pp. 411-414)

Karakas, A. I.: 2010, MNRAS 403, 1413

Matteucci, F., Calura, F.: 2005, MNRAS 360, 447

Mennekens, N., Vanbeveren, D., De Greve, J.P., De Donder, E.: 2010, A\&A 515, A89

Mushotzky, R., Loewenstein, M., Arnaud, K. A., Tamura, T., Fukazawa, Y., Matsushita, K., Kikuchi, K., Hatsukade, I.: 1996, ApJ 466, 686

Romano, D., Karakas, A. I., Tosi, M., Matteucci, F.: 2010, A\&A 522, A32

Ruiter, A. J., Belczynski, K., Fryer, C.: 2009, ApJ 699, 2026

Sato, K., Tokoi, K., Matsushita, K., Ishisaki, Y., Yamasaki, N. Y., Ishida, M., Ohashi, T.: 2007, ApJ 667, L41

Schindler, S., Diaferio, A.: 2008, SSRv 134, 363

Smith, R. K., Brickhouse, N.S., Liedahl, D. A., Raymond, J. C.: 2001, ApJ 556, L91-L95

Sullivan, M., Ellis, R. S., Howell, D. A., Riess, A., Nugent, P.E., Gal-Yam, A.: 2009, ApJ 693, L76

Sullivan, M., Le Borgne, D., Pritchet, C. J., Hodsman, A., Neill, J. D., Howell, D. A., Carlberg, R. G., et al.: 2006, ApJ 648, 868

Takahashi, T., Mitsuda, K., Kelley, R., et al.: 2010, The ASTRO-H Mission. In Proc. SPIE, Volume 7732 (p. 77320Z)

Tsujimoto, T., Nomoto, K., Yoshii, Y., Hashimoto, M., Yanagida, S., Thielemann, F. K.: 1995, MNRAS 277, 945

Vangioni, E., Silk, J., Olive, K.A., Fields, B.D.: 2011, MNRAS 413, 2987

Werner, N., Böhringer, H., Kaastra, J. S., de Plaa, J., Simionescu, A., Vink, J.: 2006a, A\&A 459, 353

Werner, N., de Plaa, J., Kaastra, J. S., Vink, J., Bleeker, J. A. M., Tamura, T., Peterson, J. R., Verbunt, F.: 2006b, A\&A 449, 475

Werner, N., Durret, F., Ohashi, T., Schindler, S., Wiersma, R. P. C.: 2008, SSRv 134, 337

Woosley, S. E., Weaver, T. A.: 1995, ApJS 101, 181 